\title{
Stability and Production of Superheavy Nuclei
}

\author{
Peter Möller*, and J. Rayford Nix ${ }^{\dagger}$ \\ * P. Moller Scientific Computing and Graphics, Inc., P. O. Box 1440, Los Alamos, New Mexico 87544, USA \\ ${ }^{\dagger}$ Theoretical Division, Los Alamos National Laboratory, Los Alamos, New Mexico 87545, USA
}

\begin{abstract}
Beyond uranium heavy elements rapidly become increasingly unstable with respect to spontaneous fission as the proton number $Z$ increases, because of the disruptive effect of the long-range Coulomb force. However, in the region just beyond $Z=100$ magic proton and neutron numbers and the associated shell structure enhances nuclear stability sufficiently to allow observation of additional nuclei. Some thirty years ago it was speculated that an island of spherical, relatively stable superheavy nuclei would exist near the next doubly magic proton-neutron combination beyond ${ }^{208} \mathrm{~Pb}$, that is, at proton number $Z=114$ and neutron number $N=184$. Theory and experiment now show that there also exists a rock of stability in the vicinity of $Z=110$ and $N=162$ between the actinide region, which previously was the end of the peninsula of known elements, and the predicted island of spherical superheavy nuclei slightly southwest of the magic numbers $Z=114$ and $N=184$. We review here the stability properties of the heavy region of nuclei.

Just as the decay properties of nuclei in the heavy region depend strongly on shell structure, this structure also dramatically affects the fusion entrance channel. The six most recently discovered new elements were all formed in cold-fusion reactions. We discuss here the effect of the doubly magic structure of the target in cold-fusion reactions on the fusion barrier and on dissipation.
\end{abstract}

\section{INTRODUCTION}

It was predicted more than 30 years ago that the next doubly magic nucleus beyond ${ }_{82}^{208} \mathrm{~Pb}_{126}$ is ${ }^{298} 114_{184}$ and that nuclei on an island of superheavy nuclei in its vicinity have half-lives of up to perhaps several billion years [1-7]. In Fig. 1 we show the calculated enhancement to binding due to microscopic effects [8] for nuclei throughout the periodic system. The two nuclei ${ }^{266} \mathrm{~Pb}$ and ${ }^{238} \mathrm{Hf}$ are so near the neutron drip line that one can anticipate that they will never be observable. Of the remaining doubly magic regions only the recently observed region near 272110 consists of nuclei deformed in their ground states. The only spherical doubly magic region that realistically remains to be observed is therefore the island of spherical superheavy elements currently predicted to occur near ${ }^{292} 114$. Although no nuclei on this superheavy island have been observed so far, six new elements with proton numbers $Z=107-112$ have been discovered between this superheavy island and the previously heaviest-known elements at the edge of the actinide region. The heaviest of the new elements are localized in the vicinity of a rock of deformed shell-stabilized nuclei near proton number $Z=110$ and neutron number $N=162$. We show in Fig. 2 the location of the currently heaviest known nucleus ${ }^{277} 112$ and its observed $\alpha$-decay products superimposed on calculated microscopic corrections for nuclei throughout the periodic system. In the following we discuss what we have learned experimentally and theoretically about (1) the stability properties of the heaviest nuclei and (2) the cold-fusion reaction mechanism that has been so essential in the discovery of the heaviest elements. A few aspects of hot fusion will also be reviewed.

\section{MODELS}

In the study of nuclear structure a substantial number of different models are used, many of which are discussed at this conference. The significance of these models and their relation to each other is made more clear if we observe that it is possible to put the nuclear models used into one of the groups below:

1. Models where the physical quantity of interest is given by an expression such as a polynomial or a more general algebraic expression. The parameters are usually determined by adjustments to experimental data. Normally models of this type describe only a single nuclear property. No nuclear wave functions are obtained in these models. An example of a model of this type is the original Bethe-Weizsäcker macroscopic mass model. A nice 
FIGURE 1. Calculated microscopic enhancement to binding for nuclei throughout the periodic system.

feature of this macroscopic mass model is that it can be generalized, without any additional parameters, to describe macroscopic fission barriers. This generalization property is unusual for models in this category.

2. Models that are claimed to be based on microscopic equations with realistic two-body interactions but which utilize so many approximations that the end result is again some algebraic expression with parameters that are adjusted to experimental data. No microscopic differential equations are actually solved. Examples of models of this type are the Duflo mass model [9] and the fermion dynamical-symmetry model [10] which is applied to nuclear mass calculations and some other calculations.

FIGURE 2. The heaviest known element ${ }^{277} 112$ and its $\alpha$-decay products as dots superimposed on calculated microscopic corrections for nuclei throughout the periodic system. 
3. Models that use an effective nuclear interaction and actually solve the resulting microscopic quantum-mechanical equations, for example a Schrödinger or a Dirac equation. The solutions provide nuclear wave functions which allow a vast number of nuclear properties to be modeled within a single framework. Most models of this type that are currently used fall into two subgroups depending on the type of wave function and the type of microscopic interaction used:

3a Single-particle models that use a simple central potential with additional residual interactions such as the two-body pairing interaction. The Schrödinger equation is solved in a single-particle approximation and additional two-body interactions are treated in the BCS, Lipkin-Nogami, or RPA approximations, for example. Wave functions are obtained, which allows a large number of nuclear-structure features to be predicted, such as transition rates within or between rotational bands or beta-decay transition rates.

For the calculation of the total nuclear energy it is not possible in the single-particle model to obtain the nuclear ground-state energy as $E=\left\langle\Psi_{0}|H| \Psi_{0}\right\rangle$, where $\Psi_{0}$ is the ground-state nuclear wave function. To obtain the nuclear potential energy as a function of shape one combines the single-particle model with a macroscopic model, which leads to the macroscopic-microscopic model in which the energy is calculated as a sum of a microscopic correction obtained from calculated single-particle levels by use of the Strutinsky method and a macroscopic energy.

3b Hartree-Fock-type models in which the postulated effective interaction is of two-body type and the wave function is an antisymmetrized Slater determinant. In other respects, these models have many similarities to those in $\mathbf{3 a}$, with the exception that it is possible to obtain the nuclear ground-state energy as $E=<\Psi_{0}|H| \Psi_{0}>$.

We sometimes hear proponents of models of type $\mathbf{3} \mathbf{b}$ refer to models of type $\mathbf{3 a}$ as "macroscopic." This is clearly inaccurate since

a both models solve microscopic wave equations. The difference between the two approaches lies in the type of interaction and type of wave function used.

b to date most if not all important new insight into microscopic nuclear structure has been provided by models in category 3a. For example, reasonably accurate ground-state deformations and masses, nuclear level structure, including spins and parities, and the mass asymmetry of the fission saddle point were first obtained in this approach. The first two model categories are only able to parameterize or polynomialize data by use of expressions that normally contain a vast number of parameters and consequently reproduce experimental

FIGURE 3. Comparison between energy releases $Q_{\alpha}$ obtained in the FRDM (1992), ETFSI-1 (1992) model, and FDSM (1992) and recent experimental data for the heaviest known element. Two different decay chains were observed experimentally. 
FIGURE 4. Nuclear shapes leading from the ground state to elongated and compact scission shapes. The corresponding potential-energy surface for ${ }^{264} \mathrm{Fm}$ is shown in Fig. 5. The quantity $R_{0}$ is the radius of the original spherical nucleus.

data used in the adjustment well, but fail catastrophically for data not used in the adjustments or for new data. Models in category $\mathbf{3} \mathbf{b}$ are expected to in principle be more accurate than models in category $\mathbf{3 a}$, because the wave function is more realistic and more realistic effective-interactions can be used. However, two problems remain today: what effective two-body interaction is realistic, in the sense that it will yield more accurate results than the well-studied and well-optimized single-particle effective interactions, and what are the optimized parameter values of such a realistic two-body interaction?

Let us further emphasize that there is no "correct" model in nuclear physics. Modeling of nuclear physics involves simplifying the true forces and equations with the goal to obtain a formulation that can be solved in practice, but that "retains the essential features" of the true system under study. How to obtain an effective force from the true

FIGURE 5. Potential-energy surface leading from the ground state to elongated and compact scission shapes. The corresponding shapes are shown in Fig. 4. 
force is not well-defined. What we mean by "retains the essential features" depends on the circumstances. Simply speaking, it means that it retains sufficiently much of the true system that we can learn something from the simplified model. Bearing this in mind, it is clear that the microscopic single-particle models have been enormously successful over the years, since we have learned so much from them.

In our brief overview here we will discuss what has been learned about the stability of the heaviest elements from calculations based on a macroscopic-microscopic model with a realistic diffuse-surface folded-Yukawa single-particle potential as a starting point for the microscopic term and a generalized droplet model with a Yukawa-plus-exponential potential for the nuclear energy in the macroscopic term. The model has been described in sufficient detail elsewhere $[8,11-14]$. For a review of other results we refer to the many other interesting talks at this conference and to a recent review $[15]$.

\section{STABILITY AT THE END OF THE PERIODIC SYSTEM}

Whereas predictions of an island of superheavy elements were made already 30 years ago, the existence of a "rock" of relatively long-lived deformed neutron-deficient shell-stabilized superheavy nuclei in the vicinity of $Z=110$ and $N=162$ has been a subject of study only for about 15 years or so. In our first global nuclear mass calculation in 1981 [13,14], which was limited to 4023 nuclei, and which did not reach the neutron and proton drip lines, part of this rock is nevertheless clearly visible in the tabulated microscopic corrections. In Fig. 1 we see that the island of superheavy elements predicted in the mid 1960s is not isolated from the relatively stable actinide region. Instead, a stabilizing peninsula extends from the spherical superheavy region towards the actinide region. On this peninsula two stabilizing ridges corresponding to $N=152$ and $N=162$ are clearly visible.

For additional clarity we have included in Figs. 1 and 2 only even-even nuclei so that odd-even staggering is removed. The ridge at $N=152$ has long been connected to the unusually long spontaneous-fission half-lives of ${ }^{250} \mathrm{Cf},{ }^{252} \mathrm{Fm}$, and ${ }^{254} \mathrm{No}[16-18]$.

The peninsula structure extending from the spherical superheavy island is somewhat similar to the smaller peninsula extending to the southwest from the doubly magic ${ }^{208} \mathrm{~Pb}$. On this peninsula the most prominent shell-stabilized ridges occur at $N=102$ and $N=108$, but are less developed than the ridges in the heavy region.

FIGURE 6. Proton single-particle level diagram for merging nuclei in an asymmetric heavy-ion collision leading to the heaviest known nucleus. The intersecting-sphere parameterization is used for the entire path from touching spheres to a single sphere. Thus, the level-diagram path does not pass exactly through the calculated ground-state shape. The asymmetric configurations in the entrance channel lead to a mixing of states with odd and even parity. The magic-fragment gaps associated with the initial entrance-channel configuration remain far inside the touching point, to about $r / R_{0}=1.15$, somewhat outside the maximum in the fusion barrier. 
In Fig. 2 we have superimposed the location of the most recently discovered heavy element ${ }^{277} 112$ and its $\alpha$-decay daughters [19] on calculated microscopic corrections obtained in the FRDM (1992) [8]. The $\alpha$-decay $Q$-values are plotted in Fig. 3 and compared to three theoretical calculations $[8,10,20,21]$. Two experimental decay chains have been observed. These decay chains provide for the first time the half-life of a nucleus, ${ }^{271} \mathrm{Hs}$, at the center of the predicted rock of stability. The measured half-life of about $10 \mathrm{~s}$ is in excellent agreement with the half-life of 630 s predicted by the FRDM (1992) [20]. The agreement between these experimental observations and theoretical predictions confirms the predictive powers of current nuclear-structure models and represents a triumph for nuclear physics.

\section{THE COLD-FUSION ENTRANCE CHANNEL}

The six heaviest-known elements were all produced in cold-fusion reactions with doubly magic ${ }_{82}^{208} \mathrm{~Pb}_{126}$ or neardoubly magic ${ }_{83}^{209} \mathrm{Bi}_{126}$ targets. The cold-fusion reaction has long been thought to enhance heavy-element evaporationresidue cross sections primarily because it leads to compound nuclei of low excitation energy, which enhances deexcitation by neutron emission relative to fission. Higher excitation energies would lead to higher fission probabilities. However, the evaporation-residue cross section is the product of the cross section for compound-nucleus formation and the probability for de-excitation by neutron emission. One may therefore ask if cold fusion also enhances the cross section for compound-nucleus formation. Because of the low excitation energies in the entrance channel, the large negative shell correction associated with target nuclei near the doubly magic ${ }^{208} \mathrm{~Pb}$ should be almost fully manifested at touching and slightly inside touching.

Nuclei near ${ }^{258} \mathrm{Fm}$ have already provided important insight into fragment shell effects in symmetric fission and fusion configurations $[15,22,23]$. At ${ }^{258} \mathrm{Fm}$ fission becomes symmetric with a very narrow mass distribution, the kinetic energy of the fragments is about $35 \mathrm{MeV}$ higher than in the asymmetric fission of ${ }^{256} \mathrm{Fm}$, and the spontaneousfission half-life is $0.38 \mathrm{~ms}$ for ${ }^{258} \mathrm{Fm}$ compared to $2.86 \mathrm{~h}$ for ${ }^{256} \mathrm{Fm}$. These features are well understood in terms of the macroscopic-microscopic model. Shell effects associated with division into fragments near ${ }^{132} \mathrm{Sn}$ lower the fusion valley at touching by about $20 \mathrm{MeV}$ in the most favorable case relative to that in macroscopic model. This fragment shell effect remains important far inside the touching point and results in fission into the fusion valley with very

FIGURE 7. Total, adiabatic, and macroscopic fusion barriers for the cold-fusion reaction ${ }^{70} \mathrm{Zn}+{ }^{209} \mathrm{Bi} \rightarrow{ }^{279} 113$ and fission barrier corresponding to spontaneous fission from the ground state. 
compact cold fragments for several fissioning nuclei in the vicinity of ${ }^{258} \mathrm{Fm}$. The maximum effect of fragment shells in symmetric fission occurs in the fission of the hypothetical nucleus ${ }^{264} \mathrm{Fm}$. In Fig. 4 we show a set of shapes leading from the nuclear ground state towards elongated scission configurations in the upper right part of the figure and to a compact scission configuration in the lower right part of the figure. The corresponding potential-energy surface calculated in the FRDM (1992) is shown in Fig. 5. The deep valley to the lower right, corresponding to cold fission into spherical fragments or to cold fusion of two ${ }^{132} \mathrm{Sn}$ nuclei, is very prominent and extends to the inner fission saddle near the ground state. The deformation coordinates $r$ and $\sigma$ correspond to the distance between the centers of mass of the two parts of the system and to the sum of the root-mean-square extensions along the symmetry axis of the mass of each half of the system about its center of mass, respectively.

We now show that shell effects are also very important in the fusion entrance channel in cold-fusion heavyion reactions, which usually involve asymmetric projectile-target combinations. In Fig. 6 we show calculated proton single-particle levels for merging, intersecting spheres in terms of the $r$ shape coordinate for the reaction ${ }^{70} \mathrm{Zn}+{ }^{208} \mathrm{~Pb} \rightarrow{ }^{278} 112$. This represents the reaction employed to reach the heaviest nucleus known thus far. We note that the magic-fragment gap combination $28+82=110$ remains far inside the touching point, up to about $r / R_{0}=1.15$. The quantity $R_{0}$ is the radius of the spherical compound system. Because of the stability of the fragment gaps during the merging of the two nuclei, excitation should be minimal until late in the fusion process, which should favor evaporation-residue formation. These results are in excellent agreement with the results of calculations related to the symmetric fission of nuclei near ${ }^{258} \mathrm{Fm}$ into symmetric spherical fragments near ${ }^{132} \mathrm{Sn}$.

To quantitatively study the effect of the persistent magic-fragment gaps on the fusion barrier as the heavy ions merge, we have calculated the fusion barrier for intersecting spheres for the proposed reaction

$$
{ }^{70} \mathrm{Zn}+{ }^{209} \mathrm{Bi} \rightarrow{ }^{279} 113
$$

which is shown in Fig. 7. Just inside the peak in the fusion barrier at about $r / R_{0}=1.0$ we have switched from the intersecting-sphere parameterization to Nilsson's perturbed-spheroid $\epsilon$ parameterization so that we accurately obtain the energy of the ground state. Such a switch is not carried out in the calculation of the level diagram, so the level-diagram path does not pass exactly through the calculated ground-state shape. The calculated ground-state shape is indicated in Fig. 7. We also show the touching configuration and one intersecting-sphere configuration at $r / R_{0}=1.0$, near the maximum in the fusion barrier. The dotted line shows the calculated fission barrier, for which we considered $\epsilon_{2}, \epsilon_{4}$, and $\epsilon_{6}$ shape distortions. The effect of mass asymmetry on the fission barrier is expected to be small. The fusion barrier in the macroscopic FRDM without any shell effects is given by the short-dashed line. The touching configuration is indicated by a thin vertical long-dashed line. The thicker long-dashed line is the calculated adiabatic barrier without any specialization energy. Despite the fairly high spin $9 / 2$ of the ${ }^{209} \mathrm{Bi}$ ground state the calculated specialization energy is quite low. The adiabatic curve is shown only from touching to about

FIGURE 8. Seven touching configurations for heavy-ion collisions with spherical projectiles and general targets. 
$r / R_{0}=1.0$. However, the specialization energy is quite low also between $r / R_{0}=1.0$ and the ground-state shape. For the cold-fusion reactions that have resulted in the formation of the elements $Z=107-112$, our calculations show that the incident energy resulting in maximum $1 \mathrm{n}$ cross section corresponds to an energy just a few $\mathrm{MeV}$ above the calculated total fusion barrier.

\section{SUPERHEAVY ELEMENTS BY HOT-FUSION REACTIONS}

When hot-fusion reactions are used to produce heavy elements, these reactions usually involve a deformed actinide target and a spherical, light projectile. The combination of a spherical projectile and deformed target is illustrated for various representative target deformations in Fig. 8. For such configurations the fusion barrier is not one-dimensional, but two-dimensional [24,25]. More generally, both the projectile and targets may be deformed. We illustrate the case of prolate, negative-hexadecapole targets and projectiles in Fig. 9. In this case the full characterization of the potential between the deformed projectile and the deformed target leads to a four-dimensional potentialenergy surface. However, major features of this multi-dimensional potential-energy surface can be obtained from a calculation of the barrier for the five limiting configurations shown in Fig. 9.

As limiting orientations we consider only situations where the projectile center is on the $x, y$, or $z$ axis of the target and orientations of the projectile where the projectile symmetry axis is either parallel to or perpendicular to the target symmetry axis. Since we restrict ourselves to axial symmetry, configurations with the projectile center located on the $x$ or $y$ axis are identical. If the projectile is located in the equatorial region of the target it can be oriented in three major orientations, and if it is located in the polar region it can be oriented in two major orientations. Thus, for a particular projectile-target deformation combination there are five possible limiting configurations. Because compact touching configurations are thought to favor compound-nucleus formation, a particularly favorable configuration could be the equatorial-cross configuration, which is the configuration shown to the right in the bottom row of Fig. 9. For the prolate, negative-hexadecapole targets and projectiles shown in this figure we call this configuration the "hugging" configuration. Another close-approach configuration would involve an oblate target and an oblate projectile in a polar-parallel configuration. The fusion-barrier configurations in deformed heavy-ion collisions are discussed in greater detail in Ref. [25].

To reach element $Z=114$ the reaction ${ }^{48} \mathrm{Ca}+{ }^{244} \mathrm{Pu} \rightarrow{ }^{292} 114$ is being considered. In Fig. 10 we show our calculated two-dimensional fusion potential-energy surface for this reaction. The target is centered at the origin. The fusion potential is shown for locations $(\rho, z)$ of the center of the projectile, where $\rho$ is the distance from the symmetry axis $z$. Since the projectile center is some distance away from the target surface when the target and projectile surfaces touch, the energy function that we use is not defined inside the gray area for the separated

FIGURE 9. Five touching configurations for heavy-ion collisions with prolate, negative-hexadecapole projectiles and targets. 
projectile-target configuration. It is immediately clear from the figure that there is a substantial difference between the polar barrier height of $193.3 \mathrm{MeV}$ and the equatorial barrier height of $208.0 \mathrm{MeV}$.

We have earlier argued that compact hugging configurations are desirable for evaporation-residue formation [25]. That is, targets and projectiles should have positive values of $\epsilon_{4}$ (negative hexadecapole moments) so that waistlines develop. This permits a close approach in the equatorial-cross orientation. Suitable targets would then be nuclei in the rare-earth region starting at approximately $Z=68$. Corresponding projectiles would then be in the range $Z=42-50$. One may, for example, consider a reaction with a spherical projectile: ${ }^{124} \mathrm{Sn}+{ }^{176} \mathrm{Yb} \rightarrow{ }^{300} 120$, for which the equatorial barrier is $378.2 \mathrm{MeV}$. This means that only two neutrons would be emitted for collisions at the barrier. An example of a true hugging configuration with negative-hexadecapole target and projectile shapes is ${ }^{114} \mathrm{Cd}+{ }^{180} \mathrm{Hf} \rightarrow{ }^{294} 120$. Here, the hugging barrier is $381.8 \mathrm{MeV}$ and the number of evaporated neutrons is four. The distance between mass centers for this configuration at touching is $11.08 \mathrm{fm}$. Reactions with oblate projectiles exhibit interesting features. An example is ${ }^{116} \mathrm{Cd}+{ }^{180} \mathrm{Hf} \rightarrow{ }^{296} 120$, for which the polar-parallel barrier is 365.6 $\mathrm{MeV}$, corresponding to a $2 \mathrm{n}$ evaporation process for collisions at the barrier. The touching distance is $11.91 \mathrm{fm}$ for this orientation. For the equatorial-transverse orientation the touching distance is only $10.49 \mathrm{fm}$, but the barrier is $389.3 \mathrm{MeV}$ for this orientation, corresponding to the emission of five neutrons.

The cold-fusion process between spherical projectiles and targets has led to the discovery of six new elements. Some features of the cold-fusion process are well understood today but other important aspects remain to be explored. In particular, we need to understand how the evaporation-residue cross section depends on the projectile and target species and on the reaction energy. The exploration of reactions between spherical or deformed projectiles and deformed targets leading to heavy elements also presents a fascinating challenge for the future. Here we need to understand also the influence of deformation and relative orientation of the target and projectile on the evaporationresidue cross section. Also, in this case one can find fairly cold reactions leading to the evaporation of relatively few neutrons. In the future, some aspects of these reactions will perhaps be explored in a radioactive-ion-beam facility with polarized targets.

FIGURE 10. Calculated potential-energy surface in units of $\mathrm{MeV}$ for the reaction ${ }^{48} \mathrm{Ca}+{ }^{244} \mathrm{Pu}$. The energy in the medium-gray area outside the target nucleus in the center was not calculated, because the points in this region correspond to points inside the touching configuration. Note the ridge with saddle points and peaks around the target. 


\section{References}

1) H. W. Meldner, unpublished (1965).

2) H. W. Meldner, Proc. Int. Symp. on why and how to investigate nuclides far off the stability line, Lysekil, 1966, Ark. Fysik 36 (1967) 593.

3) W. D. Myers and W. J. Swiatecki, Ark. Fys. 36 (1967) 343.

4) S. G. Nilsson, J. R. Nix, A. Sobiczewski, Z. Szymański, S. Wycech, C. Gustafson, and P. Möller, Nucl. Phys. A115 (1968) 545.

5) S. G. Nilsson, C. F. Tsang, A. Sobiczewski, Z. Szymański, S. Wycech, C. Gustafson, I.-L. Lamm, P. Möller, and B. Nilsson, Nucl. Phys. A131 (1969) 1.

6) J. R. Nix, Ann. Rev. Nucl. Sci. 22 (1972) 65.

7) M. Brack, J. Damgaard, A. S. Jensen, H. C. Pauli, V. M. Strutinsky, and C. Y. Wong, Rev. Mod. Phys. 44 (1972) 185 .

8) P. Möller, J. R. Nix, W. D. Myers, and W. J. Swiatecki, Atomic Data Nucl. Data Tables 59 (1995) 185.

9) J. Duflo, Nucl. Phys. A576 (1994) 29.

10) X.-L. Han, C.-L. Wu, D. H. Feng, and M. W. Guidry, Phys. Rev. C45 (1992) 1127.

11) M. Bolsterli, E. O. Fiset, J. R. Nix, and J. L. Norton, Phys. Rev. C5 (1972) 1050.

12) P. Möller and J. R. Nix, Nucl. Phys. A229 (1974) 269.

13) P. Möller and J. R. Nix, Nucl. Phys. A361 (1981) 117.

14) P. Möller and J. R. Nix, Atomic Data Nucl. Data Tables 26 (1981) 165.

15) P. Möller and J. R. Nix, J. Phys. G: Nucl. Part. Phys. 20 (1994) 1681.

16) W. J. Swiatecki, Phys. Rev. 100 (1955) 937.

17) J. Randrup, C. F. Tsang, P. Möller, S. G. Nilsson, and S. E. Larsson, Nucl. Phys. A217 (1973) 221.

18) J. Randrup, S. E. Larsson, P. Möller, S. G. Nilsson, K. Pomorski, and A. Sobiczewski, Phys. Rev. C13 (1976) 229 .

19) S. Hofmann, V. Ninov, F. P. Heßberger, P. Armbruster, H. Folger, G. Münzenberg, H. J. Schött, A. G. Popeko, A. V. Yeremin, S. Saro, R. Janik, and M. Leino, Z. Phys. A354 (1996) 229.

20) P. Möller, J. R. Nix, and K.-L. Kratz, Atomic Data Nucl. Data Tables 66 (1997) 131.

21) Y. Aboussir, J. M. Pearson, A. K. Dutta, and F. Tondeur, Atomic Data Nucl. Data Tables 61 (1995) 127.

22) P. Möller, J. R. Nix, and W. J. Swiatecki, Nucl. Phys. A469 (1987) 1.

23) P. Möller, J. R. Nix, and W. J. Swiatecki, Nucl. Phys. A492 (1989) 349.

24) P. Möller and A. Iwamoto, Nucl. Phys. A575 (1994) 381.

25) A. Iwamoto, P. Möller, J. R. Nix, and H. Sagawa, Nucl. Phys. A596 (1996) 329. 